\title{
HARDWARE ABERTO PARA INOVAÇÃO EM IOT
}

Erlon Borba de Lima (erlon.lima@ffacti.com.br) - Fundação de Apoio à Capacitação em Tecnologia da Informação - FACTI

Claudionor Pereira dos Santos (claudionor.santos@cti.gov.br) - Centro de Tecnologia da Informação Renato Archer - CTI

Roberto Ricardo Panepucci (roberto.panepucci@cti.gov.br) - Centro de Tecnologia da Informação Renato Archer - CTI

\section{RESUMO}

Este artigo descreve o desenvolvimento de soluções voltadas para uma plataforma de hardware com fonte aberta, o foco é o desenvolvimento de dispositivos para soluções IoT. Uma revisão do conceito de hardware aberto é apresentada. As oportunidades para o desenvolvimento no Brasil de módulos e componentes semicondutores são levantadas. Um 'case' para uma aplicação especifica é apresentado, visando segurança dos nós com comunicação NFC para configurações críticas. A plataforma está baseada em hardware e protocolos abertamente documentados. Os produtos deste desenvolvimento são disponibilizados na plataforma de desenvolvimento aberto colaborativo GitHub, viabilizando a rápida prototipagem de soluções, passiveis de serem integradas 'on-board' pela disponibilização aberta de esquemáticos, layouts, e documentação.

Palavras chave: Hardware Aberto, NFC, antena, Internet-of-Things

Área: Open Innovation 


\section{INTRODUÇÃO}

O conceito de Open Innovation surgiu da observação dos custos de desenvolvimento de novos produtos utilizando tecnologias cada vez mais modernas, com sofisticação e complexidade no seu desenvolvimento, que frequentemente e de forma contínua requeriam expertise além do quadro de P\&D das empresas (CHESBROUGH, 2003). A crescente necessidade de colaborar, cooperar e trocar informações exige mecanismos sofisticados para assegurar direitos e gerar valor para os parceiros envolvidos. Formalmente, o conhecimento produzido nas organizações gera valor através da proteção de mercado pela via da propriedade intelectual dos produtos que produz. Quando esse não é o caso, utilizam-se diversos mecanismos de cooperação empresarial, como por exemplo, de joint ventures e spin-offs, entre outros.

A proposta de Hardware Aberto tem uma vertente também conhecida como Libre Hardware que se baseia no modelo de 'free open source software'(FOSS). Recentemente, Pearce (2017) elaborou uma visão par um ecossistema aonde alguns modelos de negócio são propostos para agregar valor na cadeia de desenvolvimento, produção e comercialização ou serviço de hardware aberto.

O modo de operação aberto tem duas vantagens importantes: 1) permite a prototipagem rápida de inovações a partir de produtos abertos existentes; e 2) reduz substancialmente os custos de empresas com aspectos legais e jurídicos. O primeiro é o foco deste trabalho, como mostraremos, e o segundo decorre do fato de que, de um modo geral as licenças de uso são permissivas. Hoje há duas licenças aplicáveis, derivadas da experiência em software: copyleft (COPYLEFT, 2017) e permissiva (PERMISSIVA, 2017). Uma evolução é buscada por organizações como a Open Source Hardware Association, que tem os seguintes princípios (OSHW, 2017)(tradução do autor):

"Hardware de fonte aberta é hardware cujo desenho é publicamente disponibilizado de forma que qualquer um possa estudar, modificar, distribuir, produzir, e comercializar o desenho ou o hardware baseado naquele desenho. A fonte do hardware, o desenho do qual ele é feito, é disponibilizado no formato preferencial para que sejam feitas modificações a ele. Idealmente, hardware de fonte aberta utiliza componentes e materiais facilmente disponiveis, processos padrão, infraestrutura aberta, conteúdo não restrito, e ferramentas de desenho de código aberto para maximizar a capacidade de individuos de fazer e usar o hardware. Hardware de fonte aberta da às pessoas a liberdade de controlar sua tecnologia enquanto compartilhando conhecimento e promovendo comércio através da troca aberta de desenhos".

A definição do termo Hardware de Fonte Aberta é substancialmente mais elaborada e trata dos termos sobre os quais um desenho pode ser considerado legalmente aberto.

\section{O CONTEXTO BRASILEIRO}

Há no Brasil uma comunidade forte e vibrante de entusiastas de software livre e software aberto, inclusive com uma iniciativa oficial, o Software Público Brasileiro (SPB, 2017). O desenvolvimento aberto de plataformas de hardware também esta presente no país, com uma organização semelhante à OSHW (HARDWAREABERTO, 2017). Há no país uma forte presença, como no mundo todo, da plataforma conhecida como Arduíno, que permite iniciantes desenvolverem aplicações de hardware em um ambiente livremente distribuído, e 
apoiado por uma comunidade global de desenvolvedores, e fornecedores. O mercado de hardware aberto ou não para operar integrado à plataforma Arduíno é fortemente desenvolvido, com 'shields' disponíveis para inúmeras funcionalidades desejáveis para soluções IoT, tanto de comunicação em rede, como sensoreamento e atuação.

Pearce (2017) propõe que as agencias de fomento americanas implementem mecanismos de fomento científico com foco no desenvolvimento de OSHW, com cuidado para considerar o impacto em fornecedores locais. No Brasil é limitada a presença de matrizes empresariais com foco em desenvolvimento local, o que sugere considerar a adoção de políticas públicas no país, visando apoiar a adoção bem como o desenvolvimento de soluções hardware de fonte aberta. Essa estratégia pode viabilizar uma implantação de nível continental permitindo a adequação às normativas dos entes da federação aderentes às legislações, normas e regulamentos regionais, nos níveis estaduais e municipais.

Está consolidada no país uma indústria eletroeletrônica com capacidade produtiva para executar a fabricação de produtos eletroeletrônicos de forma competitiva com similares importados. Com a disponibilidade de soluções abertas, não seria necessária a aquisição de tecnologia externa, com seus 'royalties' e acordos comerciais nos moldes em prática hoje. Há de fato a necessidade de criar mecanismos que viabilizem a produção de lotes em menor escala de prototipagem a custos também reduzidos, um desafio quando comparado com a manufatura em países com alto adensamento da cadeia produtiva de eletroeletônicos.

Finalmente, a utilização de plataformas padrão, baseadas em hardware aberto na microeletrônica, para a construção de uma infraestrutura de IoT é uma estratégia nacional viável também para atingir economias de escala para componentes semicondutores. O Brasil desenvolve iniciativas no setor em várias frentes para obter esta escala, e reduzir o déficit comercial, como por exemplo a instalação da CEITEC S.A., o programa CI-Brasil, os programas FUNTEC-BNDES, e as leis de incentivo, como o PADIS, e a Lei de informática.

Neste contexto, o CTI é um centro de pesquisas e desenvolvimento que tem como missão desenvolver, aplicar e disseminar o conhecimento em TI . Possui laboratórios de projeto de circuitos integrados, sistemas de hardware, prototipagem por impressão $3 \mathrm{D}$ e de placas de circuito impresso, incluindo técnicas avançadas da indústria, como surface mount devices SMD, bem como o teste de falhas, qualificação e testes ISO 14075. O CTI é membro do programa Sibratec Microeletrônica e Eletro-Eletrônicos, além de oferecer o programa multiusuário brasileiro junto à CEITEC S.A. para a prototipagem de circuitos integrados. $\mathrm{O}$ acesso aos laboratórios é aberto, via de regra por prestação de serviços, com muitos abertos a usuários externos (CTI, 2017).

\section{MÓDULOS E COMPONENTES PARA IOT}

A atividade de desenvolvimento de soluções IoT passa por uma fase de expansão rápida aonde existe uma divisão clara nas estratégias das empresas: plataformas proprietárias; e plataformas abertas. O desenvolvimento de hardware aplicado para a integração de funções e sua disseminação em uma rede passa por avanços tecnológicos na microeletrônica. Circuitos integrados ultra-compactos, com baixo consumo, baixo custo, e funcionalidade aumentada hoje integram o sensoreamento e atuação no ambiente. A aplicação de plataformas IoT é objeto de inúmeras analogias funcionais, operacionais e transformacionais que, num extremo promete uma cornucópia de informações e oportunidades de agregar/extrair valor de dados agregados e minerados com conceitos de 'big data', e em outro extremo, são uma roupagem nova para atividades de controle e automação com compartilhamento online de dados. Esta 
enorme gama de aplicações requer uma plataforma com sensoreamento/atuação, conectividade, inteligência e programabilidade.

Plataformas padronizadas proprietárias estão sendo desenvolvidas de forma a atender de modo razoavelmente amplo, a um conjunto considerável de aplicações. Contudo, essa flexibilidade tem um overhead significante em custo, dispêndio energético, consumo de banda, e gestão.

Plataformas abertas, tem tentado oferecer uma base flexível para customização no desenvolvimento de soluções específicas para as diversas aplicações possíveis, viabilizando seu emprego com alta eficiência no consumo energético, na alocação de banda, com gestão simplificada e potencialmente baixos custos.

O custo de soluções IoT tem como um dos componentes críticos o hardware utilizado nas aplicações que demandam 'nós sensores inteligentes'. Isto está em contraponto com 'nós' tradicionais na internet, que são celulares, computadores, roteadores e demais elementos da rede que geram informações compartilhadas, como estações meteorológicas e outros serviços. Estes nós inteligente exigem customização significativa quando se considera a miríade de usos vislumbrados para diferentes instâncias de redes de 'coisas' interconectadas. Aqui existe a necessidade de introduzir o elemento de autoprovisionamento da introdução do elemento na rede, dentro de parâmetros pré-estabelecidos num planejamento.

Quando o potencial da integração oferecido por desenvolvimentos na microeletrônica "More than Moore" são considerados, o custo de cada nó tende a zero, se comparado com a função oferecida. Contudo, a economia de escala necessária para superar o 'non-recurrent engineering cost' (NRE) é um fator limitante em muitos casos. Soluções de hardware com fonte aberta que utilizem linguagens como Verilog ou VHDL para descrever sua funcionalidade, passíveis de serem implementadas em FPGAs, permitem a prototipagem de estratégias a baixo custo, para finalmente serem transferidas para silício na forma de ASICs (application specific ICs) (SIFIVE, 2017)

O objetivo a longo prazo da nossa atuação em Hardware Aberto para IoT é o desenvolvimento de soluções que requeiram hardware específico ou inovações que superem as limitações em ambientes que não atendam as especificações de soluções tradicionais, e permitam segurança no autoprovisionamento de soluções em rede.

\section{NEAR FIELD COMMUNICATIONS EM APLICAÇÕES IOT}

A configuração de determinados dispositivos IoT em campo exige cuidados especiais em se tratando da segurança da informação. A restrição de acesso físico ao sistema em campo é uma das estratégias para reduzir o risco de acesso indevido, e também de falhas na introdução e configuração de nós na rede, temas relacionados ao. Este trabalho apresenta a atividade de desenvolvimento de circuitos para configuração em campo de módulos tipo IoT, através de comunicação sem fio do tipo NFC utilizando dispositivos móveis.

A figura 1 ilustra a comunicação entre o dispositivo móvel atuando como leitor/programador do dispositivo IoT). Neste dispositivo móvel um aplicativo com código fonte aberto é utilizado para configurar o nó da rede IoT no campo. A segurança dessa comunicação é assegurada pela proximidade física com o nó, evitando o monitoramento dos parâmetros de comunicação e da configuração do equipamento. O protocolo de segurança para a 
comunicação do nó com outros nós da rede IoT e a internet é implementado internamente. (avaliar um maior detalhamento sobre a criptografia etc...)

Figura 1 - Dispositivo móvel executando programa de comunicação para configurar nó IoT

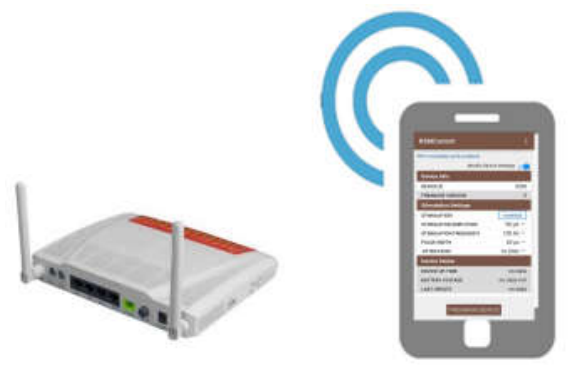

Neste 'case', a atuação com este projeto teve origem em uma sinergia na necessidade apresentada por diversos clientes (acadêmicos) que compartilhavam uma necessidade de uma configuração de hardware específica, comumente encontrada em soluções complexas de alto custo. Uma busca inicial mostrou varias iniciativa que pecavam por estarem em estágio muito inicial, ou por não possuírem documentação completa ou confiável. Concebemos então uma solução com base nas especificações do cliente, com uma visão abrangente. O projeto tomou dimensões consideráveis, e a perda do recurso humano líder do projeto resultou na terminação do mesmo. Numa fase seguinte, tomamos conhecimento da iniciativa do Prof. C. Kemere da Rice University. A figura 2 mostra uma captura da tela do site, (http://rnel.rice.edu/). Realizamos um estudo preliminar, que mostrou que as especificações poderiam atender as necessidades. Relatamos aqui a evolução da nossa contribuição ao projeto.

Figura 2 - Site do projeto com o link para seu repositório - http://rnel.rice.edu/.

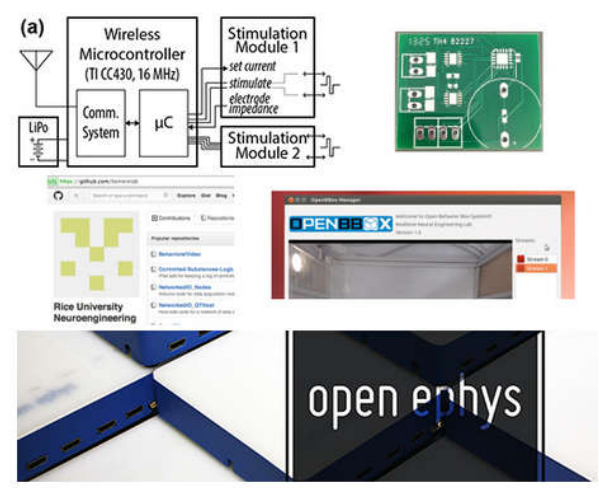

\section{Open Source Tools.}

Our lab employs standard and cutting-edge techniques: simultaneously recording the activity of dozens of neurons in behaving rodents and manipulating genetically-selected populations of neurons using light ("optogenetics"). We also are developing a number of open source software and embedded systems tools for neuroscience and neural engineering that we are eager to share. More information about tools for neuroscience and neural engineering.

\section{INOVAÇÕES NO HARDWARE DE FONTE ABERTA}

O projeto aberto obtido a partir do repositório acima foi inicialmente validado em projeto esquemático e em seguida produzido para testes. A figura 3 mostra o dispositivo tal como produzido no nosso laboratório a partir da documentação disponibilizada, e com componentes adquiridos a partir do 'bill-of-materials' (BOM). 
Figura 3. (esq) ilustração no repositório; (dir) dispositivo produzido a partir do repositório

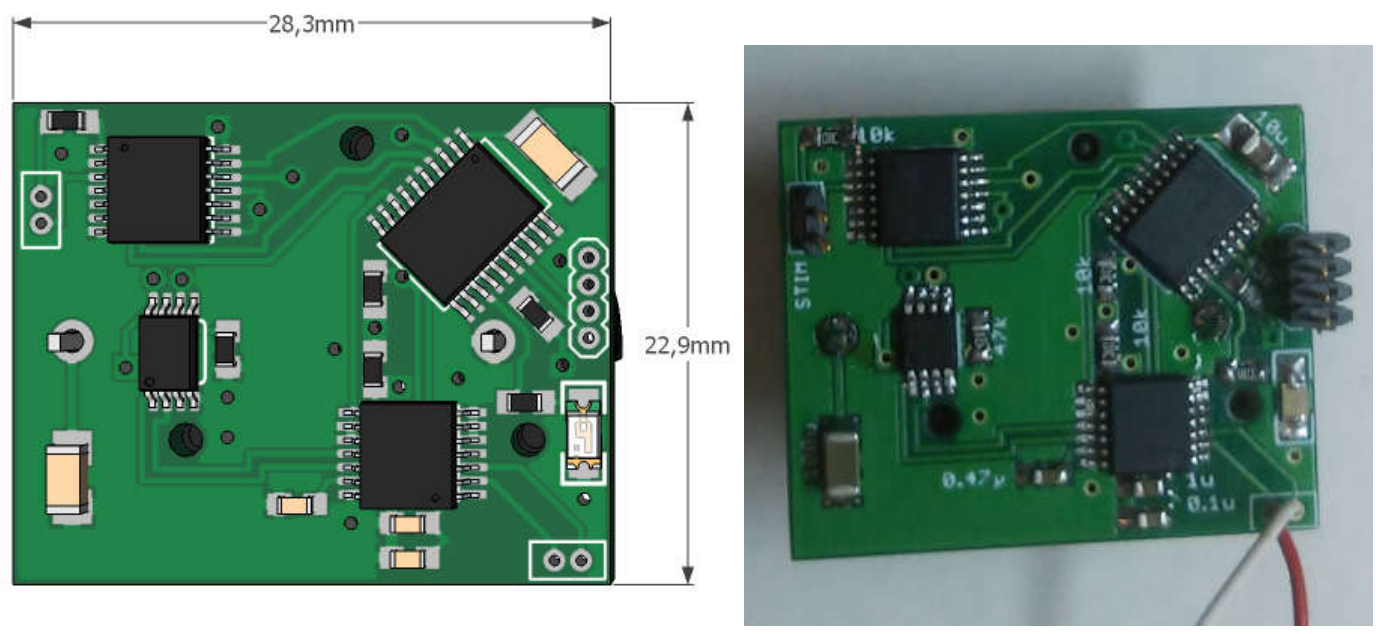

A figure 4(esq) mostra o bloco original com o circuito do CI RF430CL330H da Texas Instruments, utilizado para gerar a comunicação NFC. No projeto originalmente disponibilizado abertamente, os projetistas utilizaram a configuração do dispositivo CI RF430CL330H no modo passivo, no qual utiliza a energia coletada do estímulo de RF (radio frequência) do leitor NFC, no modo 'tag', como no caso do RFID (TEXASINSTRUMENTS 2017d), uma vez que esta configuração é muito utilizada em projetos disponíveis em repositórios de hardware aberto.

Nesta configuração passiva, operando como 'tag', a distância para iniciar e estabelecer comunicação é reduzida, o que impõe restrições ao desenho e operação do sistema. Nesse modo, o tempo de leitura e da comunicação é aumentado pela incerteza no posicionamento e em menor escala, pelo o processo de 'energy harvesting' a partir do estímulo eletromagnético do leitor requer tempo para energizar o sistema que no modo 'tag' não requer bateria. Este processo de leitura tem tempo variável dependendo de como a antena esta posicionada, da forma de manuseio, e de outros parâmetros, o que resulta em menor previsibilidade. Esta, por sua vez, requer maior atenção do operador, reduzindo a eficiência do processo, especialmente quando um grande número de operações deve ser feita.

Nossa equipe de engenheiros eletrônicos, em um espaço curto de tempo, comparado com o projeto da solução, mapeou a operação do circuito e identificou a possibilidade de aperfeiçoalo de modo significante. A solução proposta pela nossa equipe visa aumentar a distância de comunicação, e responder em um tempo mais curto e reprodutível. Isso foi possível uma vez que a aplicação específica integrava uma bateria para a função oferecida pelo módulo permitiu utilizar a configuração ativa do CI RF430CL330H com consumo em 'stand by' de 10 uA, aceitável para a aplicação em questão.

A figura 4(dir) mostra o circuito após a re-engenharia do mesmo. Nele foi introduzida a função de energizar o circuito a partir da fonte interna do dispositivo, no caso a bateria. 

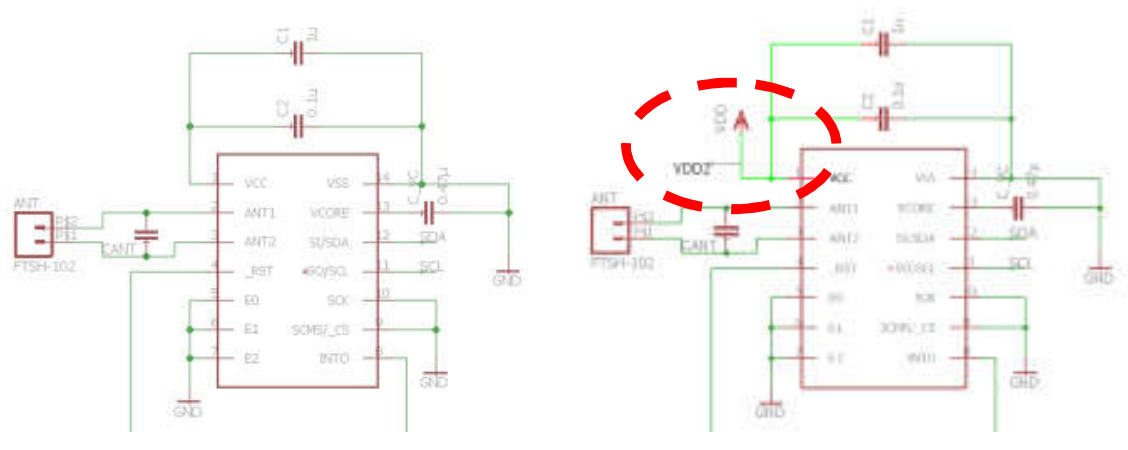

A figura 5 mostra o layout antes e depois da modificação do circuito. Esta alternativa foi comunicada diretamente ao mantenedor do projeto no repositório, e ela já consta da versão mais atualizada.

Figura 5 - Layout da placa de circuito impresso no software Eagle (esq) antes e (dir) após o aperfeiçoamento mostrado no esquemático acima.
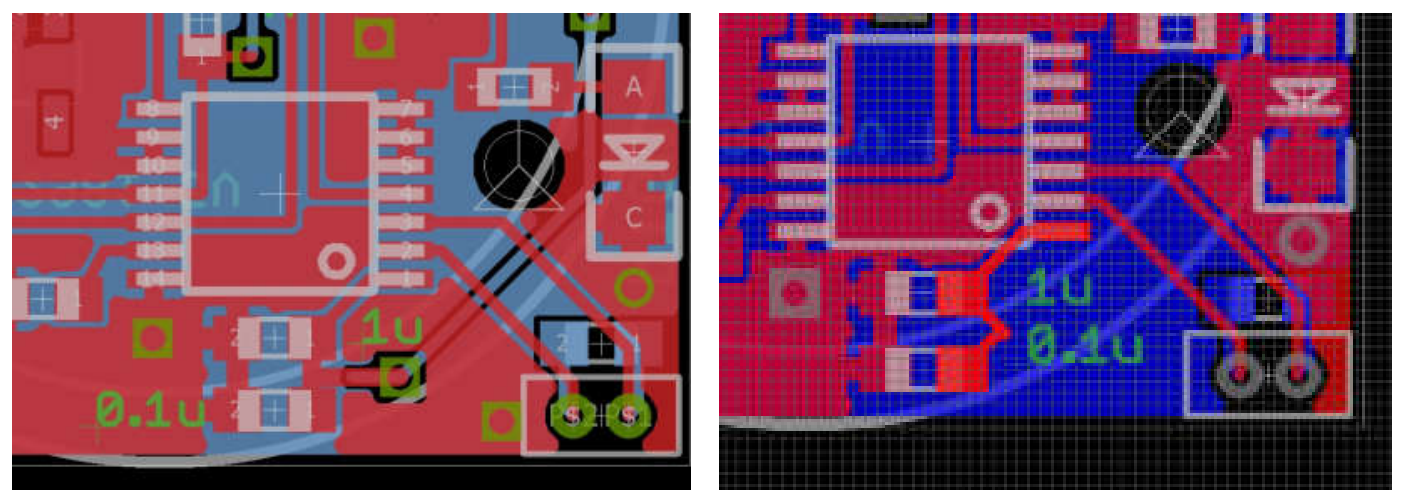

Adicionalmente, um aperfeiçoamento do projeto com foco no custo foi realizado. A oportunidade imediata de melhoria de custos foi identificada na redução do número de componentes e na complexidade de montagem. Foi reprojetado o uso de uma antena comercial, responsável por $25 \%$ do 'bill-of-materials', para comunicação por campo próximo NFC (near-field communication). A antena utilizada no projeto original é o modelo ANFCA2515-A02 da ABRACON. A proposta para reduzir o custo para o projeto deste componente foi substituída por uma solução 'built-in'.

Antenas são fundamentais para comunicação sem fio ('wireless') por rádio, e utilizam princípios do eletromagnetismo para transmitir e receber ondas eletromagnéticas nas mais diversas frequências. As dimensões físicas das antenas guardam relação próxima com o comprimento de onda do sinal de rádio utilizado, quando a propagação por distâncias maiores que este comprimento são desejadas, é o caso do sinal analógico de TV, e do rádio FM. No caso de transmissão por campo próximo para NFC este não é o caso, e as antenas na verdade atuam como dispositivos de acoplamento indutivo. A frequência de operação é de 13.56$\mathrm{MHz}$, com comprimento de onda de $20 \mathrm{~m}$. Contudo, o indutor operando no campo próximo limita detecção a poucos centímetros por dispositivos tradicionais. 


\section{RESULTADOS}

O material disponibilizado pelo fabricante foi utilizado como referencia para o design da nova antena (TexasInstruments 2017a-b). Um novo desenho foi concebido a partir das recomendações e seguindo a teoria de indutores, para permitir a menor área possível. Este desenho foi gerado juntamente com o layout do circuito todo. A figura 6 mostra uma comparação do dispositivo original e do reprojeto incluindo a antena

Figura 6 - (esq) placa original; (dir) placa com antena integrada.
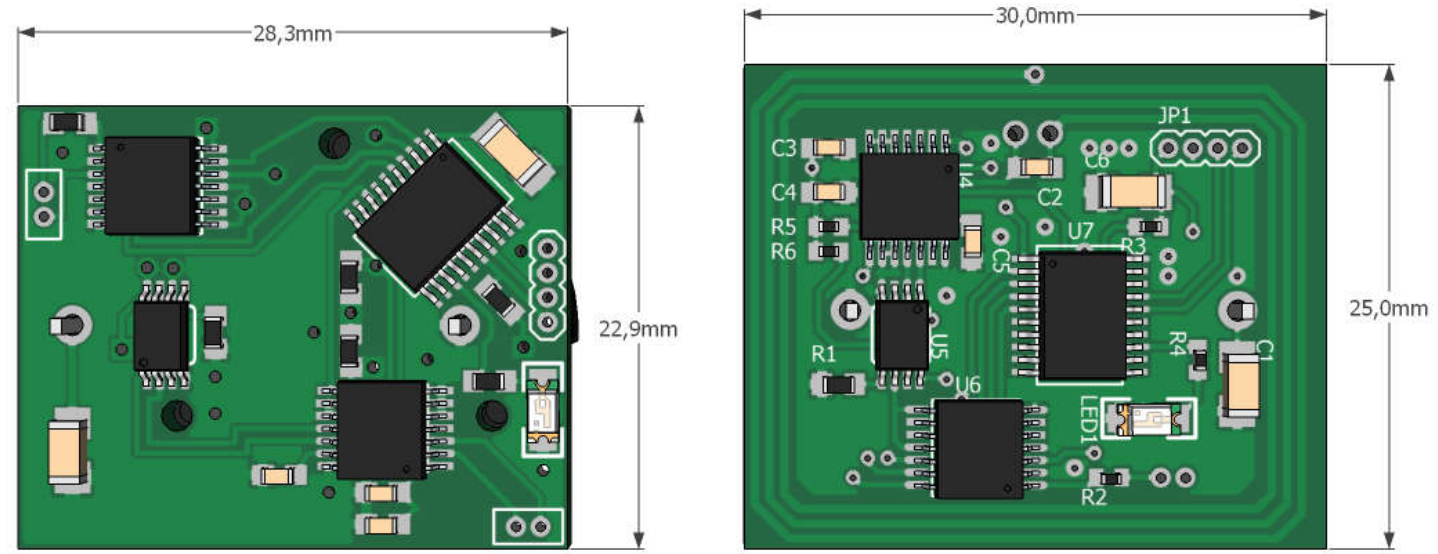

Os protótipos foram comparados no estagio de validação da antena em um mock-up simplificado, com um nível de cobre na PCB. Foi utilizado o software de projeto Eagle v8.0.0 da Autodesk ${ }^{\circledR}$. O protótipo foi construído com uma LPKF ProtoMat S103 circuit board plotter. A figura abaixo mostra os PCBs manufacturados originais e o PCB incluindo a antena. Uma área adicional de 101,93 $\mathrm{mm} 2$ (ou 17,26 \%) foi utilizada para implementar a antena. O aumento da área não causa impacto no custo da fabricação da PCB.

Figura 7 - Montagem com a tela do aplicativo no dispositivo móvel e o resultado do estímulo configurado no módulo IoT.

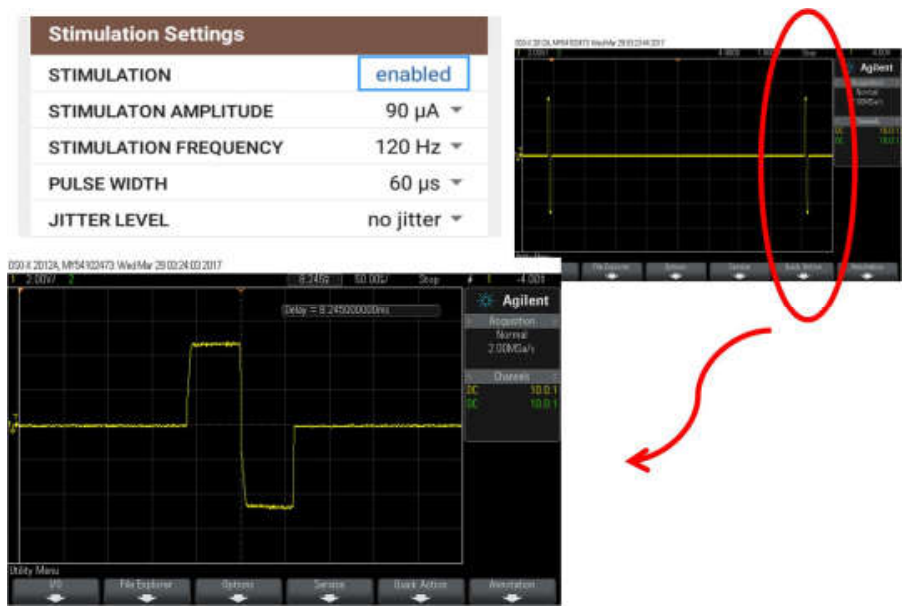

A figura 7 mostra o registro obtido na configuração. Ambas as antenas, a comercial modelo ANFCA-2515-A02 da ABRACON, e a projetada e produzida no nosso laboratório, requereram uma distância máxima entre o dispositivo móvel de leitura/escrita de aproximadamente $2 \mathrm{~cm}$, operando no modo 'tag', satisfazendo a redução de custos sem impacto na performance da solução. 


\section{CONCLUSÃO}

O estudo de uma solução NFC para aplicação em nós de rede, com potencial impacto no autoprovisionamento seguro de soluções IoT foi realizado com base em um projeto de hardware de fonte aberta. O ciclo de desenvolvimento do projeto foi de aproximadamente 30 homem-horas, facilmente entre dez a vinte vezes menor do que se desenvolvido internamente. A equipe contribuiu ao repositório para a melhoria na performance do projeto original, e obteve o conhecimento necessário para introduzir inovação no projeto interno. A demonstração da integração da antena trouxe vantagens com a redução no custo das partes e na montagem. Durante a montagem e teste do protótipo, diversas melhorias foram identificadas no circuito, que levarão a redução no consumo de energia, redução no número de componentes e portando do volume da solução.

\section{REFERÊNCIAS}

CHESBROUGH, H., Open Innovation: The New Imperative for Creating and Profiting from Technology, Harvard Business School Press, Boston, MA, 2003

CHELLA, M. T. A revolução do hardware de código aberto, Saense, 25/05/2017. URL: http://www.saense.com.br/2017/05/a-revolucao-do-hardware-de-codigo-aberto/. Acesso 05 de Junho(2017).

Copyleft. Disponível em: https://copyleft.org/. Data de acesso: 05 de Junho (2017).

CTI - http://www.cti.gov.br, Data de acesso: 05 de Junho (2017).

HARDWAREABERTO, Disponível em: https://hardwareaberto.org/wp/definicao-dehardware-aberto/ Data de acesso: 05 de Junho (2017).

OSHW, Disponível em: https://www.oshwa.org Data de acesso: 05 de Junho (2017).

PEARCE, J.M. Emerging Business Models for Open Source Hardware. Journal of Open Hardware. 1(1), p.2. DOI: http://doi.org/10.5334/joh.4 , (2017).

PERMISSIVE, Wikipedia. Permissive licence. https://en.wikipedia.org/wiki/Permissive_software_licence, Acesso: 05 de Junho (2017).

SIFIVE, processador com arquitetura RISC V open-source. https://www.sifive.com/, Data de acesso: 05 de Junho (2017).

SPB2017, https://softwarepublico.gov.br/. Data de acesso: 05 de Junho (2017).

SUMMERSON S., B. Aazhang, C. Kemere, IEEE Trans. Neural Sys. and Rehab. Eng., vol. 22, no. 6, pp. 1218, 2014; https://github.com/kemerelab/

TEXASINSTRUMENTS 2017a RF430CL330H Practical Antenna Design Guide, URL: http://www.ti.com/lit/an/sloa197/sloa197.pdf, Data de acesso: 05 de Junho (2017).

TEXASINSTRUMENTS 2017b, RF430CL330H Dynamic NFC Interface Transponder (Rev. C) URL: http://www.ti.com/lit/ds/symlink/rf430cl330h.pdf, Acesso: 05 de Junho (2017). 